Article

\title{
Atomic Norm-Based DOA Estimation with Dual-Polarized Radar
}

\author{
Min Han * (D) and Wenbin Dou \\ State Key Laboratory of Millimeter Waves, Southeast University, Nanjing 210096, China; njdouwb@163.com \\ * Correspondence: hanmin@seu.edu.cn; Tel.: +86-189-9408-3357
}

Received: 18 August 2019; Accepted: 17 September 2019; Published: 19 September 2019

\begin{abstract}
In the dual-polarized radar system, the horizontally and vertically polarized signals can be exploited to improve the direction of arrival (DOA) estimation performance. In this paper, the DOA estimation problem is considered in the dual-polarized radar. By exploiting the target sparsity in the spatial domain, the sparse-based method is proposed after formulating the DOA estimation problem as a sparse reconstruction problem. In the traditionally sparse methods using the compressed sensing (CS) theory, the spatial domain is discretized into grids to establish a dictionary matrix and solve the sparse reconstruction problem, but the off-grid error is introduced in the discretized grids. Therefore, we formulate a novel definition of atomic norm for the dual-polarized signals and give an atomic norm-based method to denoise the received signals. Then, an efficient semidefinite program (SDP) is derived, and the DOA is estimated by searching the peak values of the denoised signals. Simulation results show that the proposed method can significantly improve the DOA estimation performance in the dual-polarized radar. Additionally, compared with the state-of-art methods, the proposed method has better estimation performance with relatively low computational complexity.
\end{abstract}

Keywords: dual-polarized radar; DOA estimation; atomic norm; off-grid sparse problem

\section{Introduction}

The direction of arrival (DOA) estimation problem has been studied in applications including wireless communication, radar, and sonar and array signal processing [1-3]. Usually, the discrete Fourier transform (DFT) method is used and formulates the DOA estimation problem as a spatial sampling reconstruction problem [4-7]. However, the resolution of traditional methods is limited by the Rayleigh criterion [8,9]. To achieve a better estimation performance than the Rayleigh criterion, super-resolution methods have been proposed. The most important super-resolution methods are subspace-based methods, including the multiple signal classification (MUSIC) method [10] and the estimating signal parameters via rotational invariance techniques (ESPRIT) method [11]. Since then, extension algorithms based on the MUSIC and ESPRIT methods have been proposed in more recent papers, such as the Root-MUSIC method [12], space-time MUSIC method [13], G-MUSIC method [14], higher-order ESPRIT and virtual ESPRIT [15], etc. In the subspace-based methods, the signal and noise subspaces are estimated from the covariance of received signals, so multiple measurements are needed to obtain the corresponding covariance matrix. However, for the fast moving target, multiple measurements cannot be obtained, so the subspace-based methods cannot be used in this scenario.

To improve the DOA estimation performance, the target sparsity in the spatial domain can also be exploited [16]. Therefore, the sparse-based methods, especially the compressed sensing (CS)-based methods [17-20], were proposed to transform the DOA estimation problem into a sparse reconstruction problem. In multiple-input and multiple-output (MIMO) radar systems, a CS-based DOA estimation method was proposed in [18,21]. Additionally, in [22], a compressed sparse array scheme was proposed. 
The spatial domain is discretized into grids to obtain a dictionary matrix, and the DOA estimation problem is formulated as a sparse reconstruction problem. However, the targets can be not exactly at the discretized angles, so the discretized angles introduce the off-grid. Some off-grid methods have been proposed to solve the off-grid problem [23,24]. For example, reference [25] considered the structured dictionary mismatch and gave a sparse reconstruction method with off-grid. A sparse Bayesian inference was given in [26] with the off-grid consideration. Moreover, an iterative reweighted method estimated the off-grid and sparse signals jointly in [27]. Many papers have studied the off-grid problem. For example, in [28], the mismatch problem-including the sampling jitter in A/D conversion and model errors-was investigated and the perturbed orthogonal matching pursuit method was proposed. In [29], a new parameter-refined orthogonal matching pursuit (OMP) method was proposed to jointly estimate the off-grid positions and reflectivities of true scatterers. A new OMP-based sparse reconstruction method with parameter perturbation, named as PPOMP, was proposed in [30], in the delay-Doppler radar with the off-grid error. Additionally, in [31], a new image-focusing algorithm was given for sparsity-driven radar to image the rotating targets, and also to consider the off-grid scatterers. Moreover, in [23], the atomic norm-theory is formulated for the sparse reconstruction in the compressed sensing problem, which inspires us to address the DOA estimation problem using the atomic norm theory. However, different from the method in [23], we formulate a new type of atomic norm for the dual-polarized radar, and derive the expressions of DOA estimation. As shown in [23], the atomic norm theory is more suitable for the off-grid, underdetermined, and structured linear inverse problems based on the convex method. Therefore, we study the off-grid problem in the dual-polarized radar system based on the atomic norm theory.

In this paper, we consider the DOA estimation problem in the dual-polarized radar system. To exploit the target sparsity in the spatial domain, we formulate the DOA estimation problem as a sparse reconstruction problem. Then, we formulate a novel definition of atomic norm, which can be used to describe the sparsity of the dual-polarized signals. Based on the proposed atomic norm, we establish a denoising method for the received signal, but the denoising method is convex and cannot be solved efficiently. Therefore, a semidefinite program (SDP) optimization method is proposed to transform the nonconvex problem into a convex problem using the Schur complement theory, and can then be solved efficiently. Finally, the DOA of the target is estimated by searching the peak values of the polynomial function.

The remainder of this paper is organized as follows. The system model in the dual-polarized radar is formulated in Section 2. A novel definition of atomic norm and a DOA estimation method are given in Section 3. Simulation results are given in Section 4. Finally, Section 5 concludes the paper.

Notations: $(\cdot)^{\mathrm{H}}$ denotes the Hermitian transpose. $\|\cdot\|_{1},\|\cdot\|_{F}$, and $\|\cdot\|_{2}$ denote the $\ell_{1}$ norm, the Frobenius norm, and the $\ell_{2}$ norm, respectively. $\|\cdot\|^{*}$ denotes the dual norm. $\boldsymbol{I}_{N}$ denotes an $N \times N$ identity matrix. $\operatorname{Tr}\{\cdot\}$ denotes the trace of a matrix. $\mathcal{R}\{a\}$ denotes the real part of complex value $a$.

\section{Dual-Polarized Radar System}

In the array radar system, the dual-polarized signals are transmitted and $N$ antennas receive the echoed signals. As shown in Figure 1, we denote the horizontally polarized signal as $s_{\mathrm{H}}(t)$ and the vertically polarized signal as $s_{\mathrm{V}}(t)$. Therefore, with $K$ far-field targets, the received waveform for the horizontally polarized signal in the $n$-th antenna can be expressed as

$$
r_{\mathrm{H}, n}(t)=\sum_{k=0}^{K-1} e^{j 2 \pi(n-1) \frac{d}{\lambda} \sin \theta_{k}} \alpha_{\mathrm{H}, k} s_{\mathrm{H}}(t)+w_{\mathrm{H}, n}(t),
$$

where $d$ denotes the distance between adjacent antennas, $\lambda$ is the wavelength, and $w_{\mathrm{H}, n}(t)$ denotes the additive white Gaussian noise (AWGN) in the horizontally polarized antenna. $\theta_{k}$ is the DOA of the 
$k$-th target, and $\alpha_{\mathrm{H}, n}$ is the corresponding scattering coefficient for the horizontally polarized signal. Similarly, the received signal for the vertically polarized signal can be expressed as

$$
r_{\mathrm{V}, n}(t)=\sum_{k=0}^{K-1} e^{j 2 \pi(n-1) \frac{d}{\lambda} \sin \theta_{k}} \alpha_{\mathrm{V}, k} s_{\mathrm{V}}(t)+w_{\mathrm{V}, n}(t) .
$$

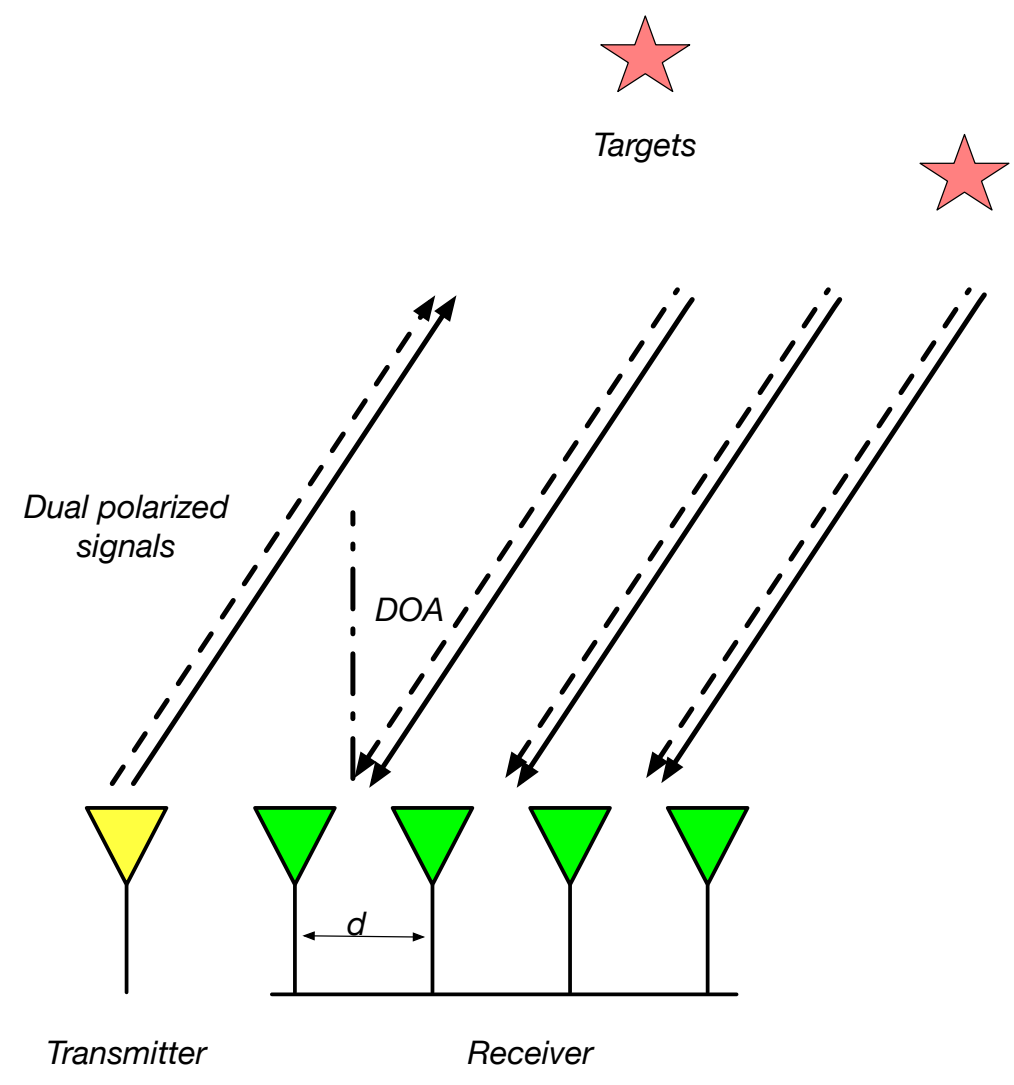

Figure 1. The dual-polarized radar system.

In this paper, we will estimate the DOA $\theta_{k}$ from the received signals $r_{\mathrm{H}, n}(t)$ and $r_{\mathrm{V}, n}(t)$. In the scenario with a fast moving target, the stationary assumption for the received signals in the antenna array no longer holds, so the traditional super-resolution methods based on the subspace decomposition, such as the MUSIC and ESPRIT methods, cannot be used in scenarios where multiple measurements are needed to obtain the covariance matrix for the stationary signals. Therefore, we consider the DOA estimation problem in the scenario with only one measurement for the polarized signals. For the horizontally polarized signal, we sample the received signals in the antennas and formulate the following vector form:

$$
r_{\mathrm{H}} \triangleq\left[r_{\mathrm{H}, 0}\left(T_{\mathrm{s}}\right), r_{\mathrm{H}, 1}\left(T_{\mathrm{s}}\right), \ldots, r_{\mathrm{H}, N-1}\left(T_{\mathrm{s}}\right)\right]^{\mathrm{T}}=\boldsymbol{A} \boldsymbol{s}_{\mathrm{H}}+\boldsymbol{w}_{\mathrm{H}}
$$

where $T_{\mathrm{S}}$ is the sampling frequency. $\boldsymbol{A}$ denotes the steering matrix $\boldsymbol{A} \triangleq\left[\boldsymbol{a}\left(\theta_{0}\right), \boldsymbol{a}\left(\theta_{1}\right), \ldots, \boldsymbol{a}\left(\theta_{K-1}\right)\right]$, and $\boldsymbol{a}(\theta)$ is the steering vector for the receiving antennas $\boldsymbol{a}(\theta) \triangleq\left[1, e^{j 2 \pi \frac{d}{\lambda} \sin \theta}, \ldots, e^{j 2 \pi \frac{(N-1) d}{\lambda} \sin \theta}\right]^{\mathrm{T}}$. $s_{\mathrm{H}} \in \mathbb{C}^{K \times 1}$, the $k$-th entry of $s$ is $\alpha_{\mathrm{H}, k} s_{\mathrm{H}}\left(T_{\mathrm{s}}\right)$ and $w_{H} \triangleq\left[w_{\mathrm{H}, 0}, w_{\mathrm{H}, 1}, \ldots, w_{\mathrm{H}, N-1}\right]^{\mathrm{T}}$. Similarly, the vertically polarized signal in the receiving antennas can be expressed as

$$
r_{\mathrm{V}} \triangleq\left[r_{\mathrm{V}, 0}\left(T_{\mathrm{s}}\right), r_{\mathrm{V}, 1}\left(T_{\mathrm{s}}\right), \ldots, r_{\mathrm{V}, N-1}\left(T_{\mathrm{s}}\right)\right]^{\mathrm{T}}=\boldsymbol{A} \boldsymbol{s}_{\mathrm{V}}+\boldsymbol{w}_{\mathrm{V}}
$$


Finally, collect all the polarized signals and we can obtain

$$
R \triangleq\left[r_{\mathrm{H}}, r_{\mathrm{V}}\right]=A S+W
$$

where $S \triangleq\left[s_{\mathrm{H}}, s_{\mathrm{V}}\right]$ and $W \triangleq\left[w_{\mathrm{H}}, w_{\mathrm{V}}\right]$. In this paper, we will estimate the DOA of target $\theta_{k}$ from the received signal $R$ using the dual-polarized radar system.

\section{Novel Atomic Norm-Based Method for DOA Estimation}

In this section, we will propose a novel atomic norm for the DOA estimation problem in the scenario with dual-polarized array radar. Based on the proposed atomic norm, an efficient method will be given to obtain the DOA using the convex optimization theory.

For the dual-polarized array radar, we formulate the following atomic norm:

$$
\begin{aligned}
\|\boldsymbol{D}\|_{\mathcal{A}} & \triangleq \inf \{d \geq 0: \boldsymbol{D} \in d \operatorname{conv}\{\mathcal{A}\}\} \\
& =\inf \left\{\|\boldsymbol{d}\|_{1}: \boldsymbol{D}=\sum_{k} d_{k} \boldsymbol{a}\left(\theta_{k}\right) \boldsymbol{t}_{k}^{\mathrm{H}}, \boldsymbol{d}=\left[d_{0}, d_{1}, \ldots d_{K-1}\right]^{\mathrm{T}},\left\|\boldsymbol{t}_{k}\right\|_{2}=1, \boldsymbol{t}_{k} \in \mathbb{C}^{2 \times 1}\right\} .
\end{aligned}
$$

Then, with the received signal $\boldsymbol{R}$, we have the following optimization problem to estimate the echoed signal:

$$
\min _{X} \frac{1}{2}\|\boldsymbol{R}-\boldsymbol{X}\|_{F}^{2}+\mu\|\boldsymbol{X}\|_{\mathcal{A}}
$$

To solve the optimization problem Equation (7), we have the following proposition.

Proposition 1. With the definition of the atomic norm in Equation (6), the optimization problem in Equation (7) can be rewritten as a dual-optimization problem:

$$
\begin{aligned}
& \min _{\boldsymbol{P}}\|\boldsymbol{R}-\boldsymbol{P}\|_{F}^{2} \\
& \text { s.t. }\|\boldsymbol{P}\|_{\tilde{\mathcal{A}}} \leq \mu,
\end{aligned}
$$

where $\|\boldsymbol{P}\|_{\tilde{\mathcal{A}}}$ is the dual norm of $\|\boldsymbol{P}\|_{\mathcal{A}}$.

Proof. The optimization problem in Equation (7) can be rewritten as

$$
\begin{aligned}
& \min _{\boldsymbol{X}} \frac{1}{2}\|\boldsymbol{R}-\boldsymbol{Z}\|_{F}^{2}+\mu\|\boldsymbol{X}\|_{\mathcal{A}} \\
& \text { s.t. } \boldsymbol{Z}=\boldsymbol{X} .
\end{aligned}
$$

Therefore, we have the Lagrange function of $\boldsymbol{X}$ and $\boldsymbol{Z}$ with the Lagrange parameter $\boldsymbol{P}$ as

$$
L(\boldsymbol{X}, \boldsymbol{Z}, \boldsymbol{P}) \triangleq \frac{1}{2}\|\boldsymbol{R}-\boldsymbol{Z}\|_{F}^{2}+\mu\|\boldsymbol{X}\|_{\mathcal{A}}+\langle\boldsymbol{Z}-\boldsymbol{X}, \boldsymbol{P}\rangle,
$$


where $\langle\boldsymbol{A}, \boldsymbol{B}\rangle \triangleq \mathcal{R}\left\{\operatorname{Tr}\left(\boldsymbol{B}^{\mathrm{H}} \boldsymbol{A}\right)\right\}$ denotes the inner product between matrices. Therefore, the dualoptimization problem of Equation (9) can be obtained as

$$
\begin{aligned}
\max _{\boldsymbol{P}} \min _{\boldsymbol{X}, \boldsymbol{Z}} L(\boldsymbol{X}, \boldsymbol{Z}, \boldsymbol{P}) & =\max _{\boldsymbol{P}} \min _{\boldsymbol{X}, \boldsymbol{Z}} \frac{1}{2}\|\boldsymbol{R}-\boldsymbol{Z}\|_{F}^{2}+\mu\|\boldsymbol{X}\|_{\mathcal{A}}+\langle\boldsymbol{Z}-\boldsymbol{X}, \boldsymbol{P}\rangle \\
& =\max _{\boldsymbol{P}}\{\min _{\boldsymbol{Z}} \underbrace{\frac{1}{2}\|\boldsymbol{R}-\boldsymbol{Z}\|_{F}^{2}+\langle\boldsymbol{Z}, \boldsymbol{P}\rangle}_{g_{1}(\boldsymbol{P}, \boldsymbol{Z})}+\min _{\boldsymbol{X}} \underbrace{\mu\|\boldsymbol{X}\|_{\mathcal{A}}-\langle\boldsymbol{X}, \boldsymbol{P}\rangle}_{g_{2}(\boldsymbol{P}, \boldsymbol{X})}\} .
\end{aligned}
$$

Since we have

$$
\begin{aligned}
\frac{\partial g_{1}(\boldsymbol{P}, \boldsymbol{Z})}{\partial \boldsymbol{Z}} & =-(\boldsymbol{R}-\boldsymbol{Z})+\frac{1}{2} \frac{\partial \operatorname{Tr}\left(\boldsymbol{P}^{\mathrm{H}} \mathbf{Z}\right)+\operatorname{Tr}^{*}\left(\boldsymbol{P}^{\mathrm{H}} \boldsymbol{Z}\right)}{\partial \mathbf{Z}} \\
& =\boldsymbol{Z}-\boldsymbol{R}+\boldsymbol{P},
\end{aligned}
$$

the $\boldsymbol{Z}$ minimizers $g_{1}(\boldsymbol{P}, \boldsymbol{Z})$ can be obtained by $\frac{\partial g_{1}(\boldsymbol{P}, \boldsymbol{Z})}{\partial \mathbf{Z}}=0$, so we have $\boldsymbol{Z}=\boldsymbol{R}-\boldsymbol{P}$. Therefore, we can obtain

$$
\min _{\boldsymbol{Z}} g_{1}(\boldsymbol{P}, \boldsymbol{Z})=\frac{1}{2}\|\boldsymbol{P}\|_{F}^{2}+\langle\boldsymbol{R}-\boldsymbol{P}, \boldsymbol{P}\rangle=\frac{1}{2}\left(\|\boldsymbol{R}\|_{F}^{2}-\|\boldsymbol{R}-\boldsymbol{P}\|_{F}^{2}\right) .
$$

With the definition of proposed atomic norm in Equation (6), we can define the dual norm of atomic norm as

$$
\|\boldsymbol{D}\|_{\tilde{\mathcal{A}}} \triangleq \sup _{\|\boldsymbol{X}\|_{\mathcal{A}} \leq 1}\langle\boldsymbol{D}, \boldsymbol{X}\rangle
$$

Therefore, we can obtain

$$
\min _{\boldsymbol{X}} g_{2}(\boldsymbol{P}, \boldsymbol{X})=-\mu\left(\max _{\boldsymbol{X}}\left\langle\boldsymbol{X}, \frac{1}{\mu} \boldsymbol{P}\right\rangle-\|\boldsymbol{X}\|_{\mathcal{A}}\right)=I\left(\|\boldsymbol{P}\|_{\mathcal{A}} \leq \mu\right),
$$

where $I(\cdot)$ is an indicate function.

Finally, the dual optimization problem can be expressed as

$$
\begin{aligned}
& \min _{\boldsymbol{P}}\|\boldsymbol{R}-\boldsymbol{P}\|_{F}^{2} \\
& \text { s.t. }\|\boldsymbol{P}\|_{\tilde{\mathcal{A}}} \leq \mu,
\end{aligned}
$$

and the proposition is proofed.

In Proposition 1, the dual-optimization problem is obtained from the atomic norm minimization (ANM) problem Equation (7), but the dual problem cannot be solved efficiently. We will show that the dual problem can be rewritten as an SDP problem. With the definition of dual norm, the constraint in Proposition 1 can be given as 


$$
\begin{aligned}
\|\boldsymbol{P}\|_{\mathcal{A}} & =\sup _{\|\boldsymbol{X}\|_{\mathcal{A}} \leq 1}\langle\boldsymbol{P}, \boldsymbol{X}\rangle \\
& =\sup _{\theta_{k} \in[0,2 \pi),\|\boldsymbol{d}\|_{1} \leq 1,\left\|\boldsymbol{t}_{k}\right\|_{2}=1}\left\langle\boldsymbol{P}, \sum_{k} d_{k} \boldsymbol{a}\left(\theta_{k}\right) \boldsymbol{t}_{k}^{\mathrm{H}}\right\rangle \\
& =\sup _{\theta \in[0,2 \pi),\|t\|_{2}=1}\left\langle\boldsymbol{P}, \boldsymbol{a}(\theta) \boldsymbol{t}^{\mathrm{H}}\right\rangle \\
& =\sup _{\theta \in[0,2 \pi),\|t\|_{2}=1} \mathcal{R}\left\{\operatorname{Tr}\left(\boldsymbol{t} \boldsymbol{a}(\theta)^{\mathrm{H}} \boldsymbol{P}\right)\right\} \\
& =\sup _{\theta \in[0,2 \pi),\|t\|_{2}=1} \mathcal{R}\left\{\boldsymbol{b}(\theta)^{\mathrm{H}} \boldsymbol{P} \boldsymbol{t}\right\} \\
& =\sup _{\theta \in[0,2 \pi)} \mathcal{R}\left\{\boldsymbol{a}(\theta)^{\mathrm{H}} \boldsymbol{P} \frac{\boldsymbol{P}^{\mathrm{H}} \boldsymbol{a}(\theta)}{\left\|\boldsymbol{P}^{\mathrm{H}} \boldsymbol{a}(\theta)\right\|_{2}}\right\} \\
& =\sup _{\theta \in[0,2 \pi)}\left\|\boldsymbol{P}^{\mathrm{H}} \boldsymbol{a}(\theta)\right\|_{2} .
\end{aligned}
$$

Hence, if we have $\sup _{\theta \in[0,2 \pi)}\left\|\boldsymbol{P}^{\mathrm{H}} \boldsymbol{a}(\theta)\right\|_{2} \leq \mu$, the constraint $\|\boldsymbol{P}\|_{\tilde{\mathcal{A}}} \leq \mu$ can be satisfied. We have the Schur complement theory as follows:

Lemma 1. For a matrix $G=\left[\begin{array}{cc}A & B \\ C & D\end{array}\right]$, we have $G \succeq 0$ if and only if we have

$$
\begin{aligned}
A & \succeq 0, \\
A-B D^{-1} C & \succeq 0 .
\end{aligned}
$$

Therefore, we can formulate a semidefinite positive matrix

$$
\left[\begin{array}{cc}
\boldsymbol{W} & \boldsymbol{P} \\
\boldsymbol{P}^{\mathrm{H}} & \mu^{2} \boldsymbol{I}
\end{array}\right] \succeq 0,
$$

so we have $\boldsymbol{W} \succeq 0$ and $\boldsymbol{W}-\mu^{-2} \boldsymbol{P} \boldsymbol{P}^{\mathrm{H}} \succeq 0$. For any vector $\boldsymbol{l}$, we have

$$
\boldsymbol{l}^{\mathrm{H}} \boldsymbol{W} \boldsymbol{l}-\mu^{-2} \boldsymbol{l}^{\mathrm{H}} \boldsymbol{P} \boldsymbol{P}^{\mathrm{H}} \boldsymbol{l} \geq 0 .
$$

When we choose $\boldsymbol{l}=\boldsymbol{a}(\theta)$, we can obtain

$$
\left\|\boldsymbol{P}^{\mathrm{H}} \boldsymbol{a}(\theta)\right\|_{2}^{2} \leq \underbrace{\boldsymbol{a}(\theta)^{\mathrm{H}} \boldsymbol{W} \boldsymbol{a}(\theta)}_{g_{3}(\boldsymbol{W}, \boldsymbol{\theta})} \mu^{2} .
$$

By letting $g_{3}(\boldsymbol{W}, \theta) \leq 1$, we can finally satisfy the constraint $\sup _{\theta \in[0,2 \pi)}\left\|\boldsymbol{P}^{\mathrm{H}} \boldsymbol{a}(\theta)\right\|_{2} \leq \mu$. We can formulate the matrix $\boldsymbol{W}$ as a Hermitian matrix, satisfying the following condition:

$$
\sum_{q} W_{q, q+k}=\left\{\begin{array}{ll}
0, & k \neq 0 \\
1, & k=0
\end{array},\right.
$$


where $W_{q, q+k}$ is the entry of $W$ at the $q$-th row and $q+k$-th column. Therefore, we can simplify $g_{3}(\boldsymbol{W}, \theta)$ as

$$
\begin{aligned}
g_{3}(\boldsymbol{W}, \theta) & =\boldsymbol{a}^{\mathrm{H}}(\theta) \boldsymbol{W} \boldsymbol{a}(\theta) \\
& =\sum_{n_{1}} \sum_{n_{2}} a_{n_{1}}^{\mathrm{H}}(\theta) a_{n_{2}}(\theta) W_{n_{1}, n_{2}} \\
& \leq 1 .
\end{aligned}
$$

Therefore, the dual optimization problem in Equation (7) can be rewritten as an SDP problem:

$$
\begin{aligned}
\min _{\boldsymbol{P}, \boldsymbol{W}}\|\boldsymbol{R}-\boldsymbol{P}\|_{F}^{2} & \begin{aligned}
\text { s.t. } & \left.\begin{array}{cc}
\boldsymbol{W} & \boldsymbol{P} \\
\boldsymbol{P}^{\mathrm{H}} & \mu^{2} \boldsymbol{I}
\end{array}\right] \succeq 0 \\
& \sum_{q} W_{q, q+k}^{n, n}= \begin{cases}0, & k \neq 0 \\
1, & k=0\end{cases}
\end{aligned}
\end{aligned}
$$

$W$ is Hermitian.

By solving this SDP problem, the denoised signal $\boldsymbol{P}$ can be obtained. Then, the DOA is estimated by the peak search of $\left\|\boldsymbol{P}^{\mathrm{H}} \boldsymbol{a}(\theta)\right\|_{2}$.

\section{Simulation Results}

In this section, the simulation results for the DOA estimation in dual-polarized radar are given, and the corresponding simulation parameters are given in Table 1. All the simulation results are obtained with $10^{4}$ Monte Carlo trials. The simulation results are carried out in a personal computer with 16 GB RAM and an Intel i7 CPU. Additionally, we compare the following state-of-art methods for the DOA estimation:

- With denoising method-this method is realized by calculating the correlation between the received signals and the steering vector to obtain the spatial spectrum, where the spatial spectrum is $\left\|\boldsymbol{R}^{\mathrm{H}} \boldsymbol{a}(\theta)\right\|\left(\theta \in\left[-60^{\circ}, 60^{\circ}\right]\right)$.

- Simultaneous orthogonal matching pursuits (SOMP) method [32]—this method is proposed for the sparse reconstruction in the scenario with multiple measurements with lower computational complexity. In the SOMP method, the column of dictionary matrix indicating the corresponding DOA is selected iteratively.

- Sparse Bayesian learning (SBL) method [26]—SBL method is the sparse Bayesian learning method and achieves good reconstruction performance in the scenario with correct distribution assumptions for the received signals, noise, and target scattering coefficients. However, the computational complexity of SBL is much higher.

Table 1. Simulation Parameters.

\begin{tabular}{cc}
\hline Parameter & Value \\
\hline The signal-to-noise ratio (SNR) of received signals & $20 \mathrm{~dB}$ \\
The number of antennas $N$ & 20 \\
The number of targets $K$ & 4 \\
The distance between adjacent antennas & 0.5 wavelength \\
The detection DOA range & {$\left[-60^{\circ}, 60^{\circ}\right]$} \\
The type of antennas & dual-polarized antennas \\
\hline
\end{tabular}


First, we show the DOA estimation for 4 targets, with the DOA being $-29.474^{\circ}, 0.68532^{\circ}$, $10.836^{\circ}$, and $30.654^{\circ}$, respectively. The spatial spectrum for the DOA estimation is shown in Figure 2, where all the methods can estimate the DOA from the received signals. The spatial spectrum of the proposed method is different from other methods, since we calculate the polynomial values $\left\|\boldsymbol{P}^{\mathrm{H}} \boldsymbol{a}(\theta)\right\|_{2}$. As shown in Equation (25), if $\theta$ is the DOA of the target, we have $\left\|\boldsymbol{P}^{\mathrm{H}} \boldsymbol{a}(\theta)\right\|_{2}=1$, so we can choose the corresponding DOA with $\left\|\boldsymbol{P}^{\mathrm{H}} \boldsymbol{a}(\theta)\right\|_{2}$ being 1 from the polynomial values. The DOA with different methods can be obtained from Figure 2, and the estimated values are given in Table 2. The DOA estimation performance is measured by the root-mean-squared error (RMSE)

$$
\mathrm{RMSE}=\sqrt{\sum_{m=0}^{M-1} \frac{\left\|\boldsymbol{\theta}_{m}-\hat{\boldsymbol{\theta}}_{m}\right\|_{2}^{2}}{M K}},
$$

where $\boldsymbol{\theta}_{m}$ denotes the target DOA in the $m$-th Monte Carlo trial, and $\hat{\boldsymbol{\theta}}_{m}$ is the estimated DOA. As shown in Table 2, the RMSEs of without denoising method, SOMP method, and SBL method are $0.21075,0.15609$, and 0.14631 in degree, respectively. The RMSE of the proposed method is 0.048264 in degree, which is much lower than existing methods. Therefore, the proposed method achieves better performance of DOA estimation.

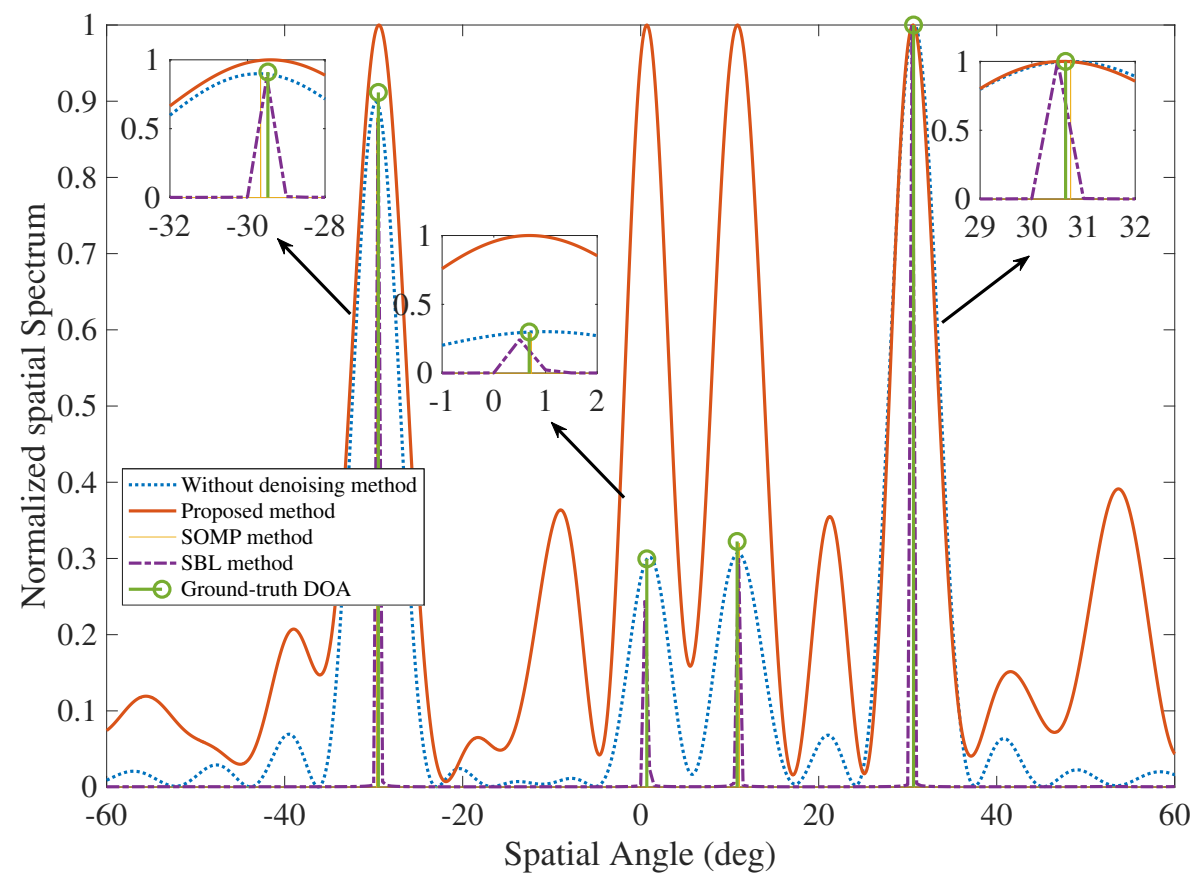

Figure 2. The spatial spectrum for direction of arrival (DOA) estimation.

Table 2. DOA Estimation Performance.

\begin{tabular}{lccccc}
\hline Methods & Target 1 & Target 2 & Target 3 & Target 4 & RMSE (deg) \\
\hline Ground-truth DOA & $-29.474^{\circ}$ & $0.68532^{\circ}$ & $10.836^{\circ}$ & $30.654^{\circ}$ & - \\
Without denoising method & $-29.723^{\circ}$ & $0.992^{\circ}$ & $10.953^{\circ}$ & $30.743^{\circ}$ & 0.21075 \\
Simultaneous orthogonal matching & $-29.663^{\circ}$ & $0.7233^{\circ}$ & $11.062^{\circ}$ & $30.75^{\circ}$ & 0.15609 \\
pursuits (SOMP) method & $-29.5^{\circ}$ & $0.5^{\circ}$ & $11^{\circ}$ & $30.5^{\circ}$ & 0.14631 \\
Sparse Bayesian learning (SBL) method & $-29.405^{\circ}$ & $0.6917^{\circ}$ & $10.876^{\circ}$ & $30.6^{\circ}$ & 0.048264 \\
\hline Proposed method & & &
\end{tabular}


Additionally, the computational complexity is shown in Table 3, where we show the computational time for one Monte Carlo trial. The without denoising method has the lowest computational complexity, with the computational time being $0.0475 \mathrm{~s}$; and that of the SOMP method is 0.6570. The SBL method has the highest computational complexity with the computational time being $6.1734 \mathrm{~s}$. The computational time of the proposed method is 2.0176, so the computational complexity is not very high and acceptable. The memory of MATLAB is $1.29 \mathrm{~GB}$ when the proposed method is running, $1.29 \mathrm{~GB}$ for the SOMP method, and 1.29 GB for the SBL method. Therefore, the memory used in the proposed method is more than the SOMP method, but almost the same as the SBL method.

Table 3. Computational Time.

\begin{tabular}{cc}
\hline Methods & Time (s) \\
\hline Without denoising method & 0.0475 \\
SOMP method & 0.6570 \\
SBL method & 6.1734 \\
Proposed method & 2.0176 \\
\hline
\end{tabular}

Then, the DOA estimation performance with different SNR in the dual-polarized radar is shown in Figure 3, where the SNR of received signals is from $0 \mathrm{~dB}$ to $40 \mathrm{~dB}$. As shown in this figure, the proposed method achieves the best DOA estimation performance, especially at SNR $\geq 15 \mathrm{~dB}$. When SNR $<15 \mathrm{~dB}$, the proposed method has almost the same performance as the SBL method, and is better than both the SOMP method and the without denoising method.

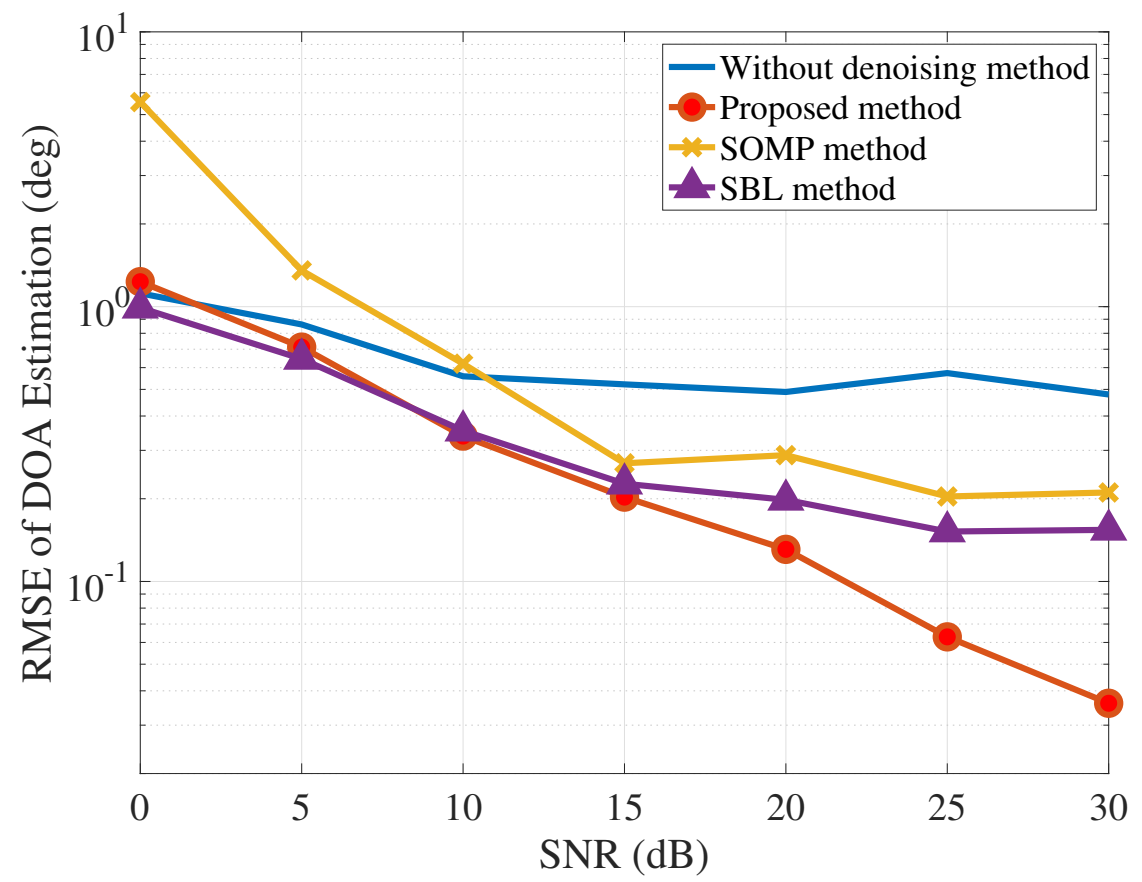

Figure 3. The DOA estimation with different SNRs.

Moreover, the DOA estimation performance with different numbers of antenna is shown in Figure 4. As shown in this figure, the DOA estimation performance is improved with increasing the number of antennas. When the antenna number is larger than 10, the proposed method achieves the best estimation performance. However, when the number of antennas is less than 10, the DOA estimation performance of the proposed method is the same as that of the SBL method.

Finally, we show the resolution performance in Figure 5, where the minimum separation is the minimum DOA difference between adjacent targets. As shown in this figure, when the separation 
is larger than $4^{\circ}$, all the methods can have better performance of DOA estimation. The performance cannot be further improved when the separation is larger than $4^{\circ}$. The proposed method has the best estimation performance with different DOA separations, and achieves the lower RMSE floor.

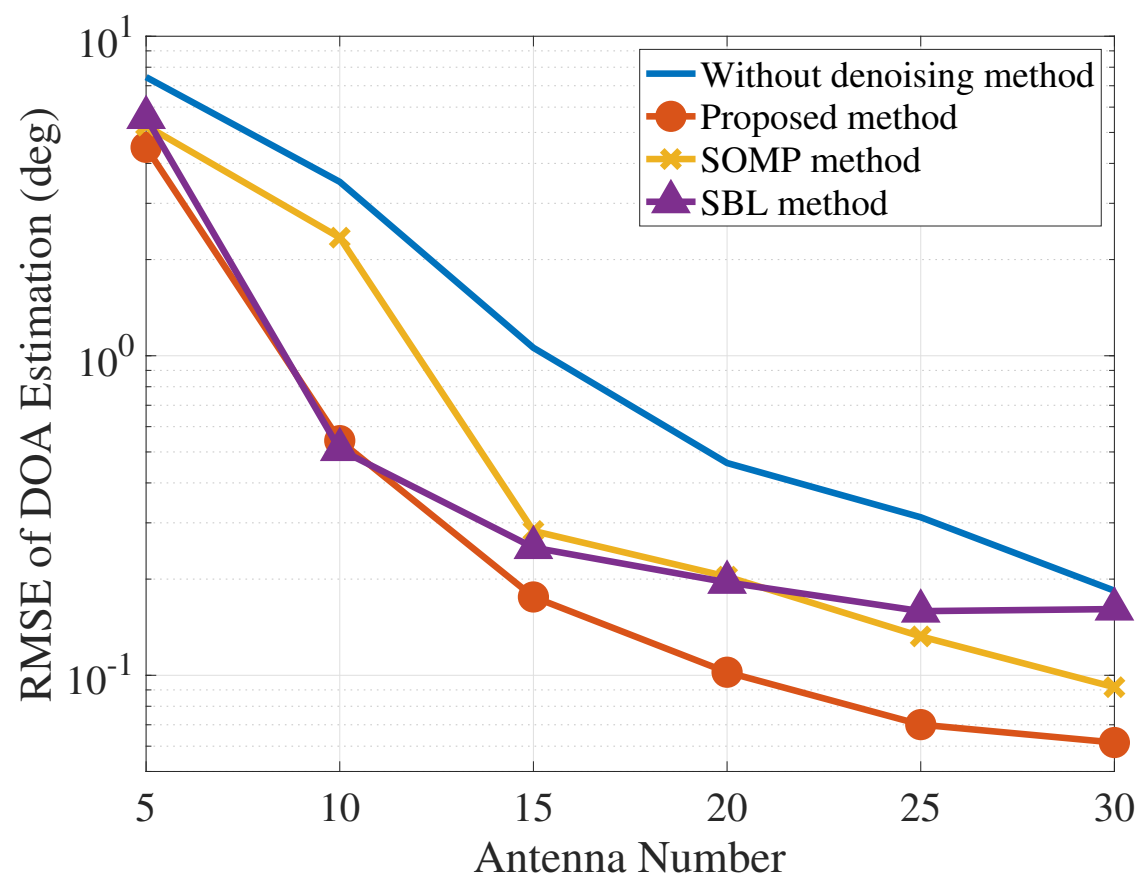

Figure 4. The DOA estimation with different numbers of antennas.

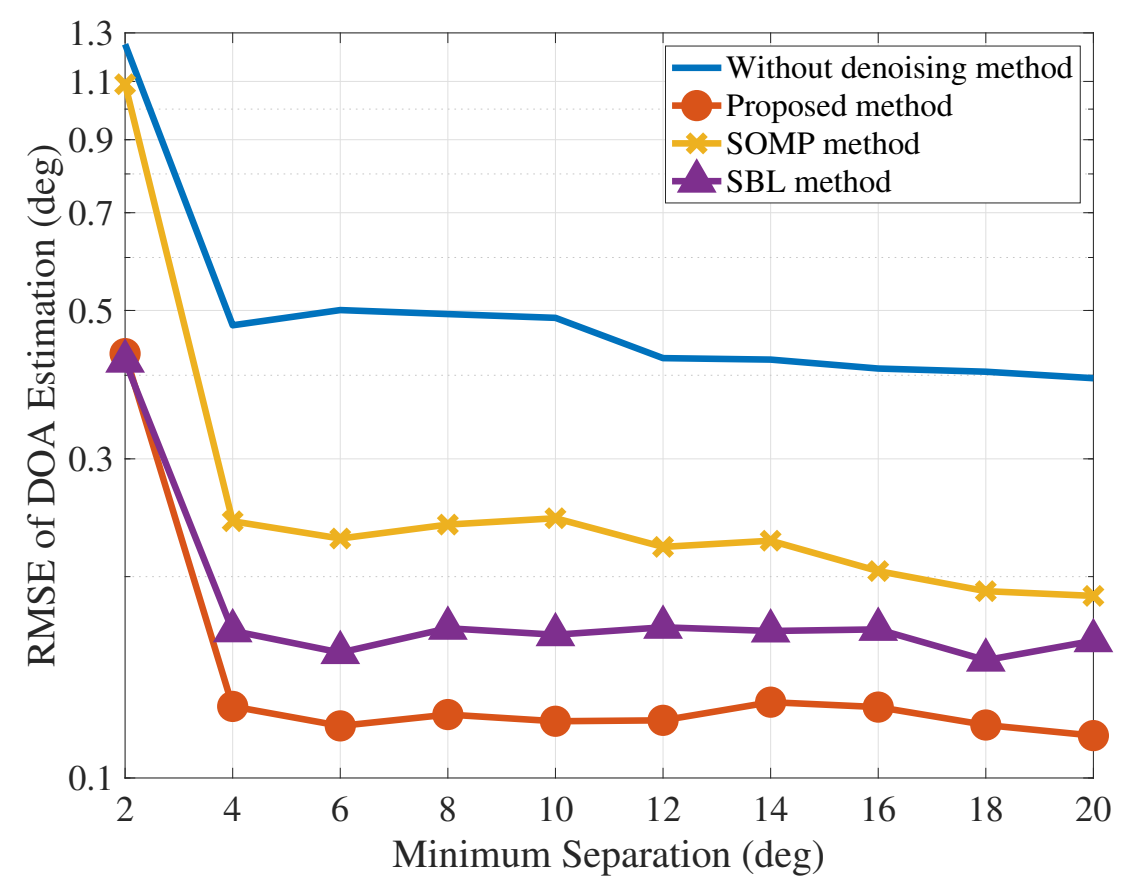

Figure 5. The DOA estimation with different DOA separations.

\section{Conclusions}

In the dual-polarized radar system, the DOA estimation problem has been addressed, and the target sparsity has been exploited to improve the DOA estimation performance. Additionally, the novel atomic norm has been defined in the scenario with dual-polarized signals, so the denoising method has 
been formulated based on the proposed atomic norm. Then, the convex SDP problem has been derived to solve the DOA estimation problem efficiently. Simulation results show that the better performance of DOA estimation for the dual-polarized signals is obtained with relatively lower computational complexity, compared with the state-of-art methods.

Author Contributions: Conceptualization, M.H. and W.D.; methodology, M.H.; software, M.H.; validation, W.D.; formal analysis, W.D.; investigation, M.H.; resources, W.D.; data curation, M.H.; writing-original draft preparation, M.H.; writing-review and editing, W.D.; visualization, M.H.; supervision, W.D.; project administration, W.D.; funding acquisition, W.D.

Funding: This work was supported by the National Natural Science Foundation of China (Grant No. 61171025)

Conflicts of Interest: The authors declare no conflict of interest.

\section{References}

1. Zheng, L.; Lops, M.; Wang, X. Adaptive Interference Removal for Uncoordinated Radar/Communication Coexistence. IEEE J. Sel. Top. Signal Process. 2018, 12, 45-60. [CrossRef]

2. Chen, P.; Cao, Z.; Chen, Z.; Liu, L.; Feng, M. Compressed Sensing-Based DOA Estimation with Unknown Mutual Coupling Effect. Electronics 2018, 7, 424. [CrossRef]

3. Cao, Z.; Geng, H.; Chen, Z.; Chen, P. Sparse-Based Millimeter Wave Channel Estimation with Mutual Coupling Effect. Electronics 2019, 8, 358. [CrossRef]

4. Burintramart, S.; Sarkar, T.; Zhang, Y.; Salazar-Palma, M. Nonconventional least squares optimization for DOA estimation. IEEE Trans. Antennas Propag. 2007, 55, 707-714. [CrossRef]

5. Kim, S.; Oh, D.; Lee, J. Joint DFT-ESPRIT estimation for TOA and DOA in vehicle FMCW radars. IEEE Antennas Wirel. Propag. Lett. 2015, 14, 1710-1713. [CrossRef]

6. Liu, L.; Liu, H. Joint estimation of DOA and TDOA of multiple reflections in mobile communications. IEEE Access 2016, 4, 3815-3823. [CrossRef]

7. Chen, P.; Qi, C.; Wu, L.; Wang, X. Waveform Design for Kalman Filter-Based Target Scattering Coefficient Estimation in Adaptive Radar System. IEEE Trans. Veh. Technol. 2018, 67, 11805-11817. [CrossRef]

8. Rueckner, W.; Papaliolios, C. How to beat the Rayleigh resolution limit: A lecture demonstration. Am. J. Phys. 2002, 70, 587. [CrossRef]

9. Chen, P.; Cao, Z.; Chen, Z.; Yu, C. Sparse off-grid DOA estimation method with unknown mutual coupling effect. Digit. Signal Process. 2019, 90,1-9. [CrossRef]

10. Schmidt, R.O. Multiple emitter location and signal parameter estimation. IEEE Trans. Antennas Propag. 1986, 34, 276-280. [CrossRef]

11. Roy, R.; Kailath, T. ESPRIT-estimation of signal parameters via rotational invariance techniques. IEEE Trans. Acoust. Speech Signal Process. 1989, 37, 984-995. [CrossRef]

12. Zoltowski, M.; Kautz, G.; Silverstein, S. Beamspace Root-MUSIC. IEEE Trans. Signal Process. 1993, 41, $344-364$. [CrossRef]

13. Claudio, E.D.D.; Parisi, R.; Jacovitti, G. Space time MUSIC: Consistent signal subspace estimation for wideband sensor arrays. IEEE Trans. Signal Process. 2018, 66, 2685-2699. [CrossRef]

14. Vallet, P.; Mestre, X.; Loubaton, P. Performance analysis of an improved MUSIC DoA estimator. IEEE Trans. Signal Process. 2015, 63, 6407-6422. [CrossRef]

15. Yuen, N.; Friedlander, B. Asymptotic performance analysis of ESPRIT, higher order ESPRIT, and virtual ESPRIT algorithms. IEEE Trans. Signal Process. 1996, 44, 2537-2550. [CrossRef]

16. Chen, P.; Chen, Z.; Zhang, X.; Liu, L. SBL-Based Direction Finding Method with Imperfect Array. Electronics 2018, 7, 426. [CrossRef]

17. Li, W.T.; Lei, Y.J.; Shi, X.W. DOA estimation of time-modulated linear array based on sparse signal recovery. IEEE Antennas Wirel. Propag. Lett. 2017, 16, 2336-2340. [CrossRef]

18. Yu, Y.; Petropulu, A.P.; Poor, H.V. Measurement matrix design for compressive sensing-based MIMO radar. IEEE Trans. Signal Process. 2011, 59, 5338-5352. [CrossRef]

19. Xiong, W.; Greco, M.; Gini, F.; Zhang, G.; Peng, Z. SFMM design in colocated CS-MIMO radar for jamming and interference joint suppression. IET Radar Sonar Navig. 2018, 12, 702-710. [CrossRef] 
20. Yang, Z.; Xie, L. Exact joint sparse frequency recovery via optimization methods. IEEE Trans. Signal Process. 2016, 64, 5145-5157. [CrossRef]

21. Chen, P.; Zheng, L.; Wang, X.; Li, H.; Wu, L. Moving target detection using colocated MIMO radar on multiple distributed moving platforms. IEEE Trans. Signal Process. 2017, 65, 4670-4683. [CrossRef]

22. Guo, M.; Zhang, Y.D.; Chen, T. DOA estimation using compressed sparse array. IEEE Trans. Signal Process. 2018, 66, 4133-4146. [CrossRef]

23. Tang, G.; Bhaskar, B.N.; Shah, P.; Recht, B. Compressed Sensing Off the Grid. IEEE Trans. Inf. Theory 2013, 59, 7465-7490. [CrossRef]

24. Chen, P.; Cao, Z.; Chen, Z.; Wang, X. Off-Grid DOA Estimation Using Sparse Bayesian Learning in MIMO Radar With Unknown Mutual Coupling. IEEE Trans. Signal Process. 2019, 67, 208-220. [CrossRef]

25. Tan, Z.; Yang, P.; Nehorai, A. Joint Sparse Recovery Method for Compressed Sensing with Structured Dictionary Mismatches. IEEE Trans. Signal Process. 2014, 62, 4997-5008. [CrossRef]

26. Yang, Z.; Xie, L.; Zhang, C. Off-grid direction of arrival estimation using sparse bayesian inference. IEEE Trans. Signal Process. 2012, 61, 38-43. [CrossRef]

27. Fang, J.; Li, J.; Shen, Y.; Li, H.; Li, S. Super-resolution compressed sensing: An iterative reweighted algorithm for joint parameter learning and sparse signal recovery. IEEE Signal Process. Lett. 2014, 21, 761-765. [CrossRef]

28. Teke, O.; Gurbuz, A.C.; Arikan, O. Perturbed Orthogonal Matching Pursuit. IEEE Trans. Signal Process. 2013, 61, 6220-6231. [CrossRef]

29. Nguyen, N.H.; Dogancay, K.; Tran, H.; Berry, P.E. Parameter-Refined OMP for Compressive Radar Imaging of Rotating Targets. IEEE Trans. Aerosp. Electron. Syst. 2019. [CrossRef]

30. Teke, O.; Gurbuz, A.C.; Arikan, O. Sparse delay-Doppler image reconstruction under off-grid problem. In Proceedings of the 2014 IEEE 8th Sensor Array and Multichannel Signal Processing Workshop (SAM), A Coruna, Spain, 22-25 June 2014; pp. 409-412.

31. Nguyen, N.H.; Doğançay, K.; Tran, H.T.; Berry, P. An Image Focusing Method for Sparsity-Driven Radar Imaging of Rotating Targets. Sensors 2018, 18, 1840. [CrossRef]

32. Determe, J.; Louveaux, J.; Jacques, L.; Horlin, F. On the Noise Robustness of Simultaneous Orthogonal Matching Pursuit. IEEE Trans. Signal Process. 2017, 65, 864-875. [CrossRef]

(C) 2019 by the authors. Licensee MDPI, Basel, Switzerland. This article is an open access article distributed under the terms and conditions of the Creative Commons Attribution (CC BY) license (http:/ / creativecommons.org/licenses/by/4.0/). 